\title{
Inhaltsverzeichnis
}

Vorwort des Herausgebers . . . . . . . . . . . . . . . IX

\section{Horst Wesser}

Methodologie der empirischen Wissenschaften als Bestandteil der Logik . . . . 1

§ 1. Logik der deduktiven und der empirischen Wissenschaften . . . . . . . 1

§ 2. Zur Struktur der Logik . . . . . . . . . . . . . . . . . . . . . . . 3

§ 3. Technische Bemerkungen . . . . . . . . . . . . . . . . . . . . . . 5

§ 4. Allgemeine Terminitheorie. . . . . . . . . . . . . . . . . . . . . . 6

$\S$ 5. Koordinaten und Bedingungen von Aussagen . . . . . . . . . . . . . 14

§ 6. Logische Explikation von Termini . . . . . . . . . . . . . . . . . . 17

§ 7. Empirische Körper . . . . . . . . . . . . . . . . . . . . . . . . . 19

§ 8. Empirische Veränderung . . . . . . . . . . . . . . . . . . . . . . 20

§ 9. Empirischer Zusammenhang . . . . . . . . . . . . . . . . . . . . 21

§ 10. Induktive Methoden . . . . . . . . . . . . . . . . . . . . . . . . 22

§ 11. Mathematisierung der empirischen Wissenschaften . . . . . . . . . . . 24

§ 12. Erklärung . . . . . . . . . . . . . . . . . . . . . . . 26

§13. Die logische Normalität der Physik . . . . . . . . . . . . . . . . . 28

§ 14. Forscher und Forschungsgegenstand . . . . . . . . . . . . . . . . . 31

§ 15. Die Normalität von Mikroobjekten . . . . . . . . . . . . . . . . . . 34

§ 16. Methodologische und physikalische Behauptungen . . . . . . . . . . . 36

\section{Johannes Dölurng}

Definitionen in empirischen Wissenschaften. . . . . . . . . . . . . . 38

§ 1. Einleitung . . . . . . . . . . . . . . . . . . . . . . . . . . . 38

§ 2. Logische Verfahren zur Bildung von Termini . . . . . . . . . . . . . 40

§ 3. Kritik einiger traditioneller Definitionsprinzipien . . . . . . . . . . . . 43

§ 4. Zur Unterscheidung von Nominal- und Realdefinitionen . . . . . . . . 45

§ 5. Definition und Bedeutungsanalyse . . . . . . . . . . . . . . . . . 47

$\S$ 6. Elementare Definitionen . . . . . . . . . . . . . . . . . . . . . . 48

$\S$ 7. Definition von Prädikaten . . . . . . . . . . . . . . . . . . 51

§ 8. Folgerungen aus Definitionen . . . . . . . . . . . . . . . . . . . 53

$\S$ 9. Implizite Definitionen . . . . . . . . . . . . . . . . . . . . . . . 56

§ 10. Operationale Definitionen . . . . . . . . . . . . . . . . . . 57

§ 11. Definition und Existenzbehauptung . . . . . . . . . . . . . . . . . 57

§ 12. Definition und Explikation . . . . . . . . . . . . . . . . . . . . . 59

§13. Schlußbemerkungen ... . . . . . . . . . . . . . . . 61 


\section{I. LoKTIONOW}

Eine funktionale Bedeutungstheorie für Termini . . . . . . . . . . . . . . 63

$\S$ 1. Inhaltliche Voraussetzungen von Theorien. . . . . . . . . . . . . . . 63

§ 2. Referentiale Bedeutung von Termini . . . . . . . . . . . . . . . . . 65

$\S$ 3. Funktionale Bedeutung von Termini . . . . . . . . . . . . . . . . . 68

$\S$ 4. Sinn (Bedeutung) von Termini . . . . . . . . . . . . . . . . . . . 68

$\S$ 5. Kontextuale Bedeutung und Synonymität . . . . . . . . . . . . . . 70

$\S$ 6. Intensionale Kontexte . . . . . . . . . . . . . . . . . . . . . . . 72

\section{HORST WeSSEL}

Modalitäten in empirischen Wissenschaften . . . . . . . . . . . . . . 77

§ 1. Modalitäten . . . . . . . . . . . . . . . . . . . . . . . . . . . . 77

§ 2. Zur Situation in der Modallogik . . . . . . . . . . . . . . . . . . . 79

§ 3. Die Modallogik von J. Eukasiewicz . . . . . . . . . . . . . . . . . . . . 80

$\S$ 4. Deutungsversuche faktischer Modalitäten und ihre Mängel . . . . . . . 88

§ 5. Die logische Struktur einfacher modaler Aussagen . . . . . . . . . . . 90

§ 6. Modalitäten und Wahrheitswerte . . . . . . . . . . . . . . . . . . 91

§ 7. Definitionsschemata zur Einführung faktischer Modalitäten . . . . . . . 92

§ 8. Definitionsschemata zur Einführung epistemischer Wissensmodalitäten . . 94

§ 9. Definitionsschemata zur Einführung deontischer Modalitäten . . . . . . . 96

§ 10. Definitionsschemata zur Einführung logischer Modalitäten . . . . . . . 97

§ 11. Absolute Modalitäten . . . . . . . . . . . . . . . . . . . . . . . . 99

§ 12. Analyse einiger Paradoxien mit Modalitäten . . . . . . . . . . . . . 99

\section{WOLERAM HeITSCH}

Fin Normenkalkül mit semantisch definierter Satzmenge. . . . . . . . . . . 108

$\S$ 1. Aufforderungen und Normen . . . . . . . . . . . . . . . . . . . . 108

$\S$ 2. Aufbau des Normenkalküls . . . . . . . . . . . . . . . . . . . . . 115

§ 3. Sätze der Normenlogik . . . . . . . . . . . . . . . . . . . . . . . 122

§ 4. Regeln des normenlogischen Schließens . . . . . . . . . . . . . . . . 126

§ 5. Konsistenz und Vollständigkeit von Normensystemen . . . . . . . . . 127

\section{Evelyn Dölling}

Einige Aspekte einer Logik empirischer Zusammenhänge . . . . . . . . . . . $\mathbf{1 3}_{\mathbf{0}}$

$\S$ 1. Einleitung . . . . . . . . . . . . . . . . . . . . . . . . . . . . 130

§ 2. Kontrafaktuale Konditionalaussagen . . . . . . . . . . . . . . . . . 132

$\S$ 3. Nomologische Aussagen . . . . . . . . . . . . . . . . . . . . . . . 133

$\S$ 4. Kausale, materiale und strikte Implikation . . . . . . . . . . . . . . 136

§ 5. Konditionale Aussagen . . . . . . . . . . . . . . . . . . . . . . . 138

$\S$ 6. Physische Folgebeziehung . . . . . . . . . . . . . . . . . . . 140

§ 7. Einwirkung . . . . . . . . . . . . . . . . . . . . . . . . . . . . 142

§ 8. Ursache. . . . . . . . . . . . . . . . . . . . . . . . . . . . . 144

§ 9. Arten von Kausalzusammenhängen. . . . . . . . . . . . . . . . . . 145

$\S$ 10. Determinismus und Indeterminismus . . . . . . . . . . . . . . . . . 147

§11. Schlußbemerkungen . . . . . . . . . . . . . . . . . . . . . 147 


\section{H. WESSEL/K. WUTTICH}

Ein System der epistemischen Logik . . . . . . . . . . . . . . . . . . . 150

$\S$ 1. Intuitive Grundlagen der epistemischen Logik . . . . . . . . . . . . . . 150

§ 2. Axiomatischer Aufbau des Systems $S E^{1}$. . . . . . . . . . . . . . . . 154

§ 3. Verschiedene Typen von Sprechern . . . . . . . . . . . . . . . . . 155

$\S$ 4. Widerspruchsfreiheit des Systems $S E^{1}$. . . . . . . . . . . . . . . . 157

$\S$ 5. Unabhängigkeit des Systems $S E^{\mathbf{1}}$. . . . . . . . . . . . . . . . . . . . 159

\section{KLaUs Wuttich}

Logische Explikationen von Informiertheits- oder Wissensaussagen . . . . . . . 164

$\S$ 1. Das System $S E I^{1}$. . . . . . . . . . . . . . . . . . . . . . . . . 165

§ 2. Widerspruchsfreiheit des Systems $S E I^{1}$ und einige Metatheoreme . . . . . 166

$\S$ 3. Unabhängigkeit des Systems $S E I^{1} \quad$. . . . . . . . . . . . . . . . . . . . . 169

$\S$ 4. Vollständigkeit des Systems $S E I^{1}$. . . . . . . . . . . . . . . . . . 171

\& 5. Das System $S E I^{1 *}$. . . . . . . . . . . . . . . . . . . . . . . . . . . . 173

$\S$ 6. Die Systeme $S E I^{2 *}, S E I^{3 *}, S E I^{2}$ und $S E I^{3}$. . . . . . . . . . . . . . . . . . 174

§ 7. Außerlogische Forderungen an die Sprecher . . . . . . . . . . . . . . 176

§ 8. Regeln für Sprecher, die mit logischen Regeln bekannt sind . . . . . . . . . 180

\section{WERNER StrazNer}

Grundbegriffe einer Theorie der Diskussion und epistemische Logik . . . . . . . 187

$\S$ 1. Grundprinzipien der Theorie der Diskussion . . . . . . . . . . . . . . . 188

§ 2. Klassische Logik und epistemische Logik . . . . . . . . . . . . . . . . . 194

§ 3. Synonymität in epistemischen Kontexten . . . . . . . . . . . . . . . . 198

$\S$ 4. Monologische und polylogische Systeme der Behauptungslogik $S^{e i}$ und $S^{v i}$. 200

\section{A. A. IwIN}

Wahrheit und Zeit . . . . . . . . . . . . . . . . . . . . 207

\section{WOLF KUMMER}

Statistische Wahrscheinlichkeit und logische Hypothesenwahrscheinlichkeit . . . 224

$\S$ 1. Versuchsschemata und Versuche . . . . . . . . . . . . . . . . . . . 224

$\S$ 2. Relative Häufigkeiten . . . . . . . . . . . . . . . . . . . . . . . 227

§ 3. Das Problem der Existenz von Wahrscheinlichkeiten . . . . . . . . . . 228

$\S$ 4. Forderungen an einen adäquaten Begriff der statistischen Wahrscheinlichkeit 229

§. Kritik einiger Auffassungen zum Wahrscheinlichkeitsbegriff . . . . . . . 231

§. Gibt es einen adäquaten Begriff der statistischen Wahrscheinlichkeit? . . . . 236

§ 7. Zur Wahrscheinlichkeit von Hypothesen . . . . . . . . . . . . . . . 239

§ 8. Zum logischen Wahrscheinlichkeitsbegriff von Carnap . . . . . . . . . 241

\section{M. SLOtNIKOW}

Erkenntnistheoretische Analyse der objektiven Basis der Physik . . . . . . . 247

\section{A. Ch. Muchamedow}

Zur sprachlichen Situation in der modernen Physik . . . . . . . . . . . . . 271

$\S$ 1. Der unkorrekte Gebrauch von Gedankenexperimenten . . . . . . . . . 271

§ 2. Quantenmechanischer Versuch und klassisches Gedankenexperiment . . . 274 
§ 3. Die qualitativen Besonderheiten des Systemverhaltens . . . . . . . . 277

§ 4. Zum Verhältnis von Logik und Empirie in der Physik . . . . . . . . . 281

$\S$ 5. Pseudodefinitionen in der modernen Physik . . . . . . . . . . . . . . 284

G. A. KUSNEZOW

Zur logischen Grundlegung der speziellen Relativitätstheorie und der Quanten-

mechanik . . . . . . . . . . . . . . . . . . . . . . . . . . . . . . . 289

$\S$ 1. Definition einiger Hilfstermini . . . . . . . . . . . . . . . . . . . . 290

§ 2. Zeit . . . . . . . . . . . . . . . . . . . . . . . . . . 291

$\S 3$. Raum . . . . . . . . . . . . . . . . . . . . . . . . . . . . . . 296

$\S$ 4. Zur Lorentz-Transformation. . . . . . . . . . . . . . . . . . . . . 306

$\S$ 5. Zur Schrödinger-Gleichung . . . . . . . . . . . . . . . . . 315

W. N. DEMIDENKo

Logische Analyse einiger Probleme der Elektrodynamik . . . . . . . . . . . 317

$\S$ 1. Analyse der logischen Grundlagen physikalischer Theorien. . . . . . . . 317

$\S$ 2. Was bringt die Materialisation des Photons? . . . . . . . . . . . . . 321

Autorenverzeichnis . . . . . . . . . . . . . . . . . . . . 337

Personenregister ....................... 339 